\title{
Diagnostic Accuracy of a Novel On-site Virtual Fractional Flow Reserve Parallel Computing System
}

\author{
Hyung-Bok Park ${ }^{1,2 *}$, Yeonggul Jang ${ }^{1 *}$, Reza Arsanjani ${ }^{3}$, Minh Tuan Nguyen ${ }^{4}$, Sang-Eun Lee ${ }^{1,5}$, \\ Byunghwan Jeon ${ }^{1}$, Sunghee Jung ${ }^{1}$, Youngtaek Hong ${ }^{1}$, Seongmin $\mathrm{Ha}^{1}$, \\ Sekeun Kim ${ }^{1}$, Sang-Wook Lee ${ }^{4}$, and Hyuk-Jae Chang ${ }^{1,5}$ \\ ${ }^{1}$ Connect-AI Research Center, Yonsei University College of Medicine, Seoul, Korea; \\ ${ }^{2}$ Department of Cardiology, International St. Mary's Hospital, Catholic Kwandong University College of Medicine, Incheon, Korea; \\ ${ }^{3}$ Mayo Clinic, Division of Cardiology, Department of Internal Medicine, Scottsdale, AZ, USA; \\ ${ }^{4}$ School of Mechanical Engineering, University of Ulsan, Ulsan, Korea; \\ ${ }^{5}$ Division of Cardiology, Severance Cardiovascular Hospital, Yonsei University Health System, Seoul, Korea.
}

Purpose: To evaluate the diagnostic accuracy of a novel on-site virtual fractional flow reserve (vFFR) derived from coronary computed tomography angiography (CTA).

Materials and Methods: We analyzed 100 vessels from 57 patients who had undergone CTA followed by invasive FFR during coronary angiography. Coronary lumen segmentation and three-dimensional reconstruction were conducted using a completely automated algorithm, and parallel computing based vFFR prediction was performed. Lesion-specific ischemia based on FFR was defined as significant at $\leq 0.8$, as well as $\leq 0.75$, and obstructive CTA stenosis was defined that $\geq 50 \%$. The diagnostic performance of vFFR was compared to invasive FFR at both $\leq 0.8$ and $\leq 0.75$.

Results: The average computation time was 12 minutes per patient. The correlation coefficient (r) between vFFR and invasive FFR was 0.75 [95\% confidence interval (CI) 0.65 to 0.83 ], and Bland-Altman analysis showed a mean bias of 0.005 (95\% CI - 0.011 to 0.021 ) with $95 \%$ limits of agreement of -0.16 to 0.17 between vFFR and FFR. The accuracy, sensitivity, specificity, positive predictive value, and negative predictive value were $78.0 \%, 87.1 \%, 72.5 \%, 58.7 \%$, and $92.6 \%$, respectively, using the FFR cutoff of 0.80 . They were $87.0 \%, 95.0 \%, 80.0 \%, 54.3 \%$, and $98.5 \%$, respectively, with the FFR cutoff of 0.75 . The area under the receiver-operating characteristics curve of vFFR versus obstructive CTA stenosis was 0.88 versus 0.61 for the FFR cutoff of 0.80 , respectively; it was 0.94 versus 0.62 for the FFR cutoff of 0.75 .

Conclusion: Our novel, fully automated, on-site vFFR technology showed excellent diagnostic performance for the detection of lesion-specific ischemia.

Key Words: Fractional flow reserve, myocardial; computed tomography angiography; patient-specific computational modeling

Received: August 21, 2019 Revised: December 4, 2019

Accepted: December 19, 2019

Corresponding author: Sang-Wook Lee, PhD, School of Mechanical Engineering, University of Ulsan, 93 Daehak-ro, Nam-gu, Ulsan 44610, Korea.

Tel: 82-52-259-2765, Fax: 82-52-259-1680, E-mail: leesw@ulsan.ac.kr

*Hyung-Bok Park and Yeonggul Jang contributed equally to this work.

-The authors have no potential conflicts of interest to disclose.

(c) Copyright: Yonsei University College of Medicine 2020

This is an Open Access article distributed under the terms of the Creative Commons Attribution Non-Commercial License (https://creativecommons.org/licenses/ by-nc/4.0) which permits unrestricted non-commercial use, distribution, and reproduction in any medium, provided the original work is properly cited.

\section{INTRODUCTION}

Fractional flow reserve (FFR) is currently the gold standard for detecting lesion-specific myocardial ischemia..$^{1-3}$ Prior studies have demonstrated that ischemia-causing lesions detected using FFR portend poor prognosis. ${ }^{1}$ Furthermore, FFR-guided percutaneous coronary intervention (PCI) has shown survival benefits over invasive coronary angiography (ICA)-guided PCI alone. ${ }^{2,3}$

Coronary computed tomography angiography (CTA) is a noninvasive method for accurate detection and exclusion of 
high-grade coronary stenoses, hence it may serve as a gatekeeper for invasive catheterization. ${ }^{4-7}$ However, CTA tends to overestimate the severity of coronary artery stenosis, resulting in low specificity and positive predictive values (PPVs)., ${ }^{8,9}$

Non-invasive CTA-derived FFR, which applies computational fluid dynamics (CFD) onto three-dimensional (3D) coronary lumen geometry derived from CTA, has demonstrated high diagnostic accuracy, as well as improved specificity and PPVs, according to three prospective multicenter trials. ${ }^{10-12} \mathrm{Ul}-$ timately, this novel technique allows for comprehensive anatomic and physiologic diagnosis of coronary artery disease (CAD).${ }^{13}$ However, current non-invasive FFR techniques have several major limitations when applied to day-to-day clinical practice: The required simulation has to be performed outside of the hospital because it necessitates a supercomputer, therefore requiring transfer of patient data potentially leading to exposure of personal patient information. ${ }^{14}$ Furthermore, the processing time can take several hours, and the service can be expensive. $^{14-18}$

Therefore, we aimed to develop a new non-invasive on-site vFFR computing system derived from CTA that would allow for instantaneous utilization in clinical practice that is timelier and cost efficient. This technique uses a novel parallel computing method with a completely automated lumen segmentation algorithm without the need for a supercomputer.

\section{MATERIALS AND METHODS}

\section{Study population}

We consecutively enrolled clinically stable adult patients from September 2015 to February 2016 at Severance Cardiovascular Hospital who underwent clinically indicated ICA within 30 days following CTA with no intervening coronary events. Institutional Review Board (Severance Hospital, IRB Number 1-2017-0031) approval was obtained for this retrospective study and informed consent was waived. Patients were excluded if they met any of the following criteria: history of coronary artery bypass graft surgery; prior PCI with suspected instent restenosis; old myocardial infarction; complex congenital heart disease; prior pacemaker or defibrillator; prosthetic heart valve; significant arrhythmia; body mass index greater than 40; or evidence of active clinical instability or life-threatening disease.

\section{CTA data acquisition}

CTA images were acquired using two 64-slice multi-detector row computed tomography systems (Somatom Sensation 64, Siemens Medical Solutions, Forchheim, Germany) with prospective or retrospective electrocardiographic gating. All patients with a heart rate of 65 beats per minute or higher received $100 \mathrm{mg}$ of atenolol orally prior to the CT, unless contraindicated. In addition, all patients received a 0.3-mg sublingual dose of nitroglycerin just prior to scanning, unless contraindicated. Bolus tracking was used for contrast injection. The scan parameters for the machines were as follows: $64 \times 0.6-\mathrm{mm}$ section collimation and a 330-ms rotation time. Depending on body habitus, tube voltages and currents were adjusted as follows: 80,100 , or $120 \mathrm{kVp}$ and $150-500 \mathrm{mAs}$. The median radiation dose was $3.92 \mathrm{mSv}$ [95\% confidence interval (CI) 2.56 to 5.65], with 9 patients receiving less than $1 \mathrm{mSv}$.

\section{Invasive FFR measurement}

Fractional flow reserve was measured in vessels deemed clinically indicated for evaluation. After administration of nitroglycerin, a pressure-monitoring guidewire was advanced distal to a lesion. Hyperemia was induced by administration of intravenous or intracoronary adenosine at a rate of $140 \mathrm{mg} / \mathrm{kg} / \mathrm{min}$, and FFR was calculated by dividing the mean distal coronary pressure by the mean aortic pressure during hyperemia. FFR at a threshold of $\leq 0.80$ or $\leq 0.75$ were considered hemodynamically significant leading to ischemia.

\section{Fully automated lumen segmentation algorithm}

We reconstructed patient-specific coronary geometry automatically using in-house software on CTA images (Fig. 1A). The fully automatic segmentation was performed with the following four steps: at first, aorta and ostia were detected by our algorithm, which uses a Bayesian formulation in a pairwise fashion using anatomical and geometrical information. ${ }^{19}$ The detected points were used as seeds for coronary artery tracking. Second, the centerlines of the left and right coronary arteries (RCA) were extracted starting from the detected ostia by our vessel tracking method based on stochastic geometric processes using an active branch search. ${ }^{20}$ The tracking method could better find the branches, any stenotic lesions, and seemingly disconnected vessels that may be occluded by atherosclerotic plaque by modeling the statistical branch occurrence and the vessel disconnection. Third, luminal boundaries for all vessels were automatically delineated every cross-sectional plane based on the image gradient and prior CT information on the number of plaques. Finally, we generated the surface mesh of the coronary structure with the estimated luminal boundaries. The connections of bifurcated vessels were processed smoothly using a mesh merging method that merged the vessel mesh and the divaricating branch mesh. ${ }^{21}$

\section{Computational fluid dynamics for vFFR}

To calculate vFFR, 3D blood flow dynamics were simulated using the CFD technique for patient-specific coronary geometry (Fig. 1B). The continuity and Navier-Stokes equations for unsteady incompressible Newtonian fluid flow were solved with appropriate boundary conditions. A fully implicit four-step fractional method with a regular Crank-Nicolson scheme for time advancement and a P2P1 finite element method for spatial discretization were employed. In order to accommodate 


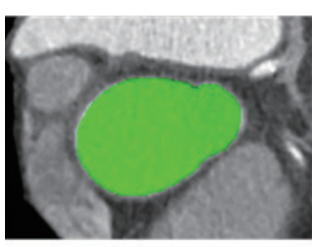

Aorta segmentation

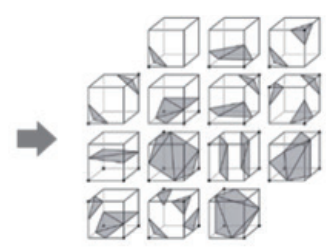

Marching cube method

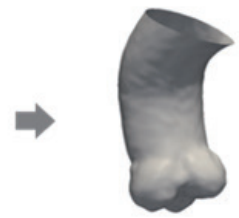

Aorta mesh

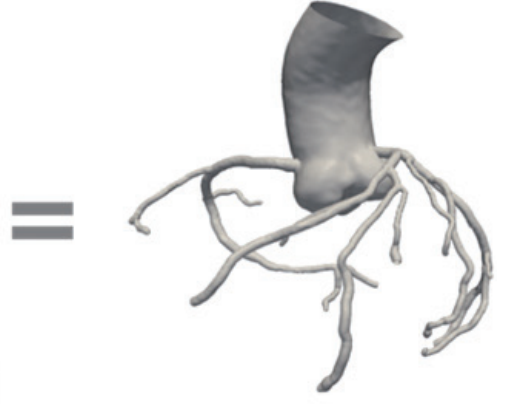

Merged mesh=coronary+aorta

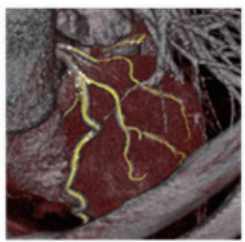

Vessel tracking

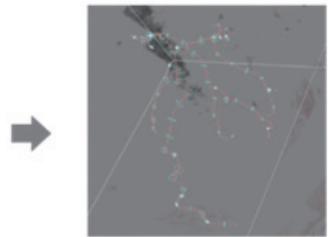

Lumen detection

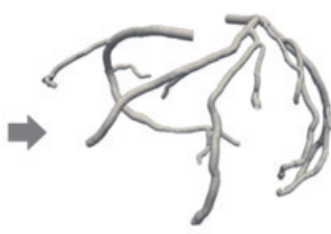

Coronary mesh

A
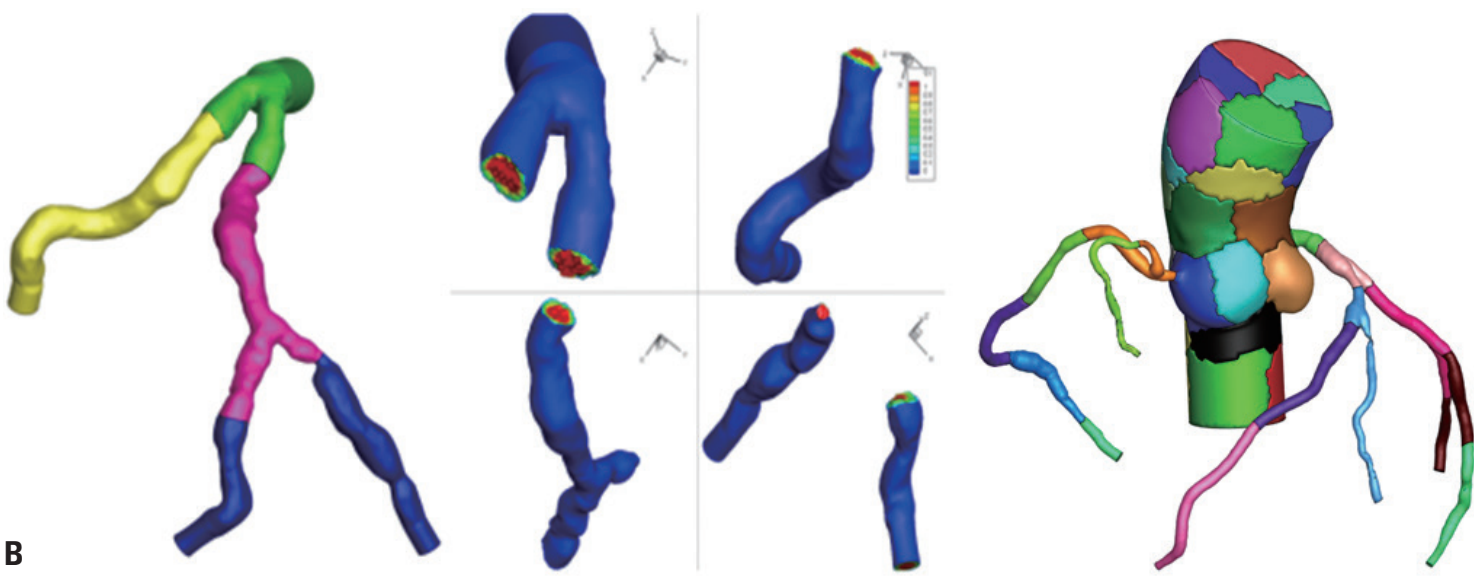

Fig. 1. Workflow of the automated segmentation algorithm and the novel parallel computing method. (A) A fully automated lumen segmentation algorithm was applied to reconstruct patient-specific coronary geometry. (B) A novel parallel computing procedure based on a cluster with 40 cores decomposing the domain into 40 sub-domains and assigning a sub-domain to each computing core was applied.

the specific time-varying pressure-flow characteristics of coronary flow, which is affected by intramyocardial pressure subject to cyclic contraction of the left ventricle and microcirculation in coronary distal networks, zero dimensional lumped parameter network (LPN) models were integrated into the 3D CFD model at all coronary outlets in a fully implicit manner. The patient-specific total resistances and capacitance for the distal coronary bed in a resting condition, as well as a hyperemic condition (i.e., the maximum flow condition resulting from the minimal microvascular resistance), were determined as described by Sankaran, et al. ${ }^{22}$ and Sharma, et al. ${ }^{23}$ The distribution of flow rates between left anterior descending artery (LAD) and left circumflex artery (LCX) was determined according to the total volume of the corresponding vessel tree. Flow division into further distal branches was approximated by allometric scaling laws based on the coronary lumen cross-sectional area. ${ }^{24}$ The aortic pressure and flow rate were acquired by solving the LPN models with constraints that matched the systolic and the diastolic blood pressure of the individual patients.

To expedite simulation time, a parallel computing procedure based on a domain decomposition method was applied (Fig. 1B). ${ }^{25}$ The P2P1 unstructured mesh of the whole computational domain was decomposed into multiple subdomains using a $k$-way partition method, ${ }^{26}$ and the computing work for each subdomain was equally distributed to corresponding processors. Simulations were carried out on a parallel cluster system (two nodes, 40 cores), where each node comprised two CPUs (E5-2680V2, $2.8 \mathrm{GHz}, 25 \mathrm{MB}$ L3 cache) and 64 GB RAM, with an Infiniband interconnect network between nodes.

\section{Statistical analysis}

Data are reported as a mean \pm SD for continuous variables and as proportions (\%) for categorical variables. Pearson correlation coefficients using two-sided $p$-values, with $p$-values $<0.05$ considered statistically significant, and Fisher's Z transformation statistics were applied for comparing correlations. Bland- 
Altman plots with 95\% CIs for correlations were calculated. FFRs were each dichotomized at a threshold of 0.80 and 0.75 , with FFR values $\leq 0.80$ and $\leq 0.75$, respectively, considered hemodynamically significant and causal of ischemia. The diagnostic accuracy, sensitivity, specificity, PPV, and negative predictive value (NPV) between vFFR and FFR at 0.80 and 0.75 were presented as proportions and 95\% CIs. The areas under receiver operating characteristic curves (AUC) were compared according to the method proposed by DeLong, et al. ${ }^{27}$ Statistical analyses were performed using Medcalc software (version 17.6; MedCalc Software, Mariakerke, Belgium).

\section{RESULTS}

Three out of 60 patients analyzed (5\%) were excluded based on failure of lumen segmentation due to severely calcified lesions or motion artifacts. We subsequently analyzed 100 lesions from 57 patients ( 42 men and 15 women, mean age: $67.3 \pm 8.5$ years). Baseline patient characteristics are presented in Table 1. The

Table 1. Baseline Characteristics of the Study Population

\begin{tabular}{|c|c|}
\hline \multicolumn{2}{|c|}{ Baseline characteristics ( $n=57$ ) } \\
\hline Mean age, yrs & $67.3 \pm 8.5$ \\
\hline Male, \% & 73.7 \\
\hline Mean body-mass index & $25.1 \pm 2.7$ \\
\hline Hypertension, \% & 56.1 \\
\hline Diabetes, \% & 31.6 \\
\hline Dyslipidemia, \% & 45.6 \\
\hline Family history, \% & 7 \\
\hline Current smoker, \% & 29.8 \\
\hline \multicolumn{2}{|l|}{ Vital signs } \\
\hline Systolic blood pressure, $\mathrm{mm} \mathrm{Hg}$ & $135.9 \pm 15.5$ \\
\hline Diastolic blood pressure, $\mathrm{mm} \mathrm{Hg}$ & $77.0 \pm 10.0$ \\
\hline Heart rate, beat/min & $60.7 \pm 8.9$ \\
\hline \multicolumn{2}{|l|}{ Laboratory measures } \\
\hline Hemoglobin, mg/dL & $13.9 \pm 1.5$ \\
\hline Hematocrit, \% & $41.0 \pm 4.5$ \\
\hline Creatinine, $\mathrm{mg} / \mathrm{dL}$ & $0.89 \pm 0.22$ \\
\hline Total cholesterol, mg/dL & $159.5 \pm 39.8$ \\
\hline LDL cholesterol, mg/dL & $88.7 \pm 33.3$ \\
\hline HDL cholesterol, mg/dL & $44.6 \pm 11.7$ \\
\hline Triglycerides, mg/dL & $124.1 \pm 79.6$ \\
\hline \multicolumn{2}{|l|}{ Medications, \% } \\
\hline Aspirin & 50.9 \\
\hline Clopidogrel & 28.1 \\
\hline Beta-blocker & 26.3 \\
\hline Nitrate & 19.3 \\
\hline Statins & 57.9 \\
\hline ACE inhibitors/ARB & 22.8 \\
\hline Calcium channel blocker & 36.8 \\
\hline
\end{tabular}

LDL, low-density lipoprotein; HDL, high-density lipoprotein; ACE, angiotensinconverting enzyme; ARB, angiotensin II receptor blocker. lesions were primarily located in the LAD (47\%), followed by the RCA (23\%), LCX (18\%), diagonal branch (DG, 5\%), obtuse marginal branch (OM, 4\%), left main (LM, 2\%), and ramus intermedius (RI, 1\%). The plaque composition profiles of the lesions were as follows: mixed plaque (59\%), non-calcified plaques (29\%), and calcified plaques (12\%). Forty percent of plaques were located in the proximal segments of coronary arteries.

The average time for generating patient-specific 3D coronary geometry was 233 seconds ( $\min =175 \mathrm{~s}, \mathrm{Q} 1=196 \mathrm{~s}$, median=212 s, $\mathrm{Q} 3=221 \mathrm{~s}, \max =386 \mathrm{~s}$ ). CFD simulations were carried out up to two cardiac cycles to damp initial transients and most runs were able to be completed within 20 minutes (average $=514.7 \mathrm{~s}$, $\min =232 \mathrm{~s}, \mathrm{Q} 1=380 \mathrm{~s}$, median=439 s, Q3=544 s, max=1370 s). The run time was highly dependent on the smoothness of coronary lumen surface and computational mesh quality. The representative simulation case of vFFR is shown in Fig. 2.

The correlation coefficient between vFFR and FFR was 0.75 (95\% CI 0.65 to 0.83 ), and Bland-Altman analysis reavealed a mean bias of 0.005 (95\% CI -0.011 to 0.021 ), with $95 \%$ limits of agreement of -0.16 to 0.17 between vFFR and FFR (Fig. 3). Diagnostic performance was evaluated separately using FFR cutoffs of 0.80 and 0.75 . The accuracy, sensitivity, specificity, PPV, and NPV when the FFR cutoff of 0.80 was used were $78.0 \%(95 \%$ CI 64.0-87.0), 87.1\% (95\% CI 70.2-96.4), 72.5\% (95\% CI 60.482.5), 58.7\% (95\% CI 48.6-68.1), and 92.6\% (95\% CI 83.2-96.9), respectively. In addition, the AUC value for vFFR was significantly higher $[0.88$ (95\% CI 0.80-0.94) vs. 0.61 (95\% CI 0.510.71 )] than CTA $\geq 50 \%$ stenosis (Fig. $4 \mathrm{~A}$ ). Moreover, when a FFR cutoff of 0.75 was used the diagnostic performance significantly improved, with accuracy, sensitivity, specificity, PPV,

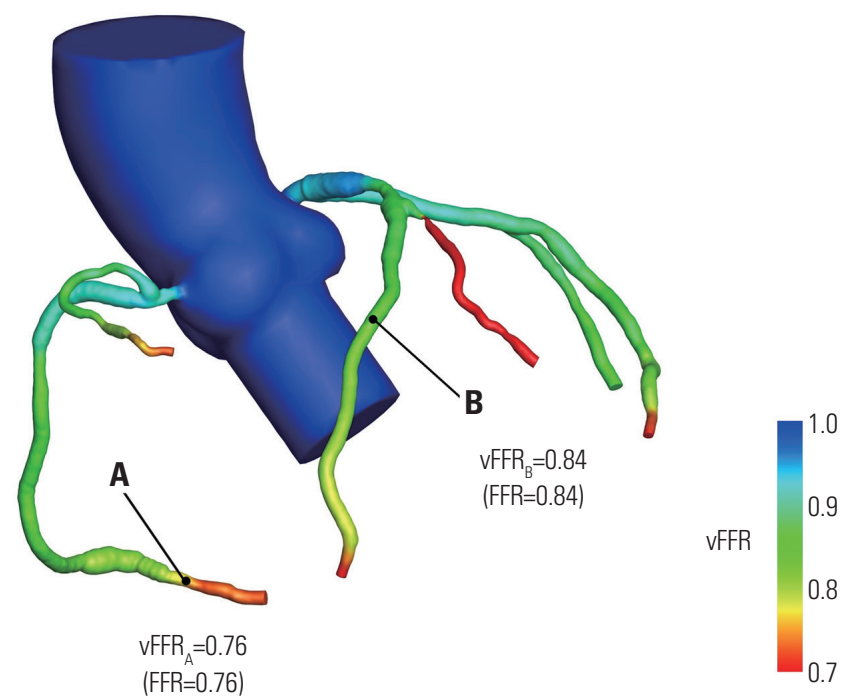

Fig. 2. Example simulation case of on-site virtual fractional flow reserve (vFFR). A noninvasive on-site vFFR simulation defined the distal portion of the right coronary artery $(A)$ as an ischemic lesion $(0.76)$ and the middle portion of the left anterior descending artery $(B)$ as a non-ischemic lesion (0.84). These simulation derived values matched perfectly with the invasively measured FFR values. 

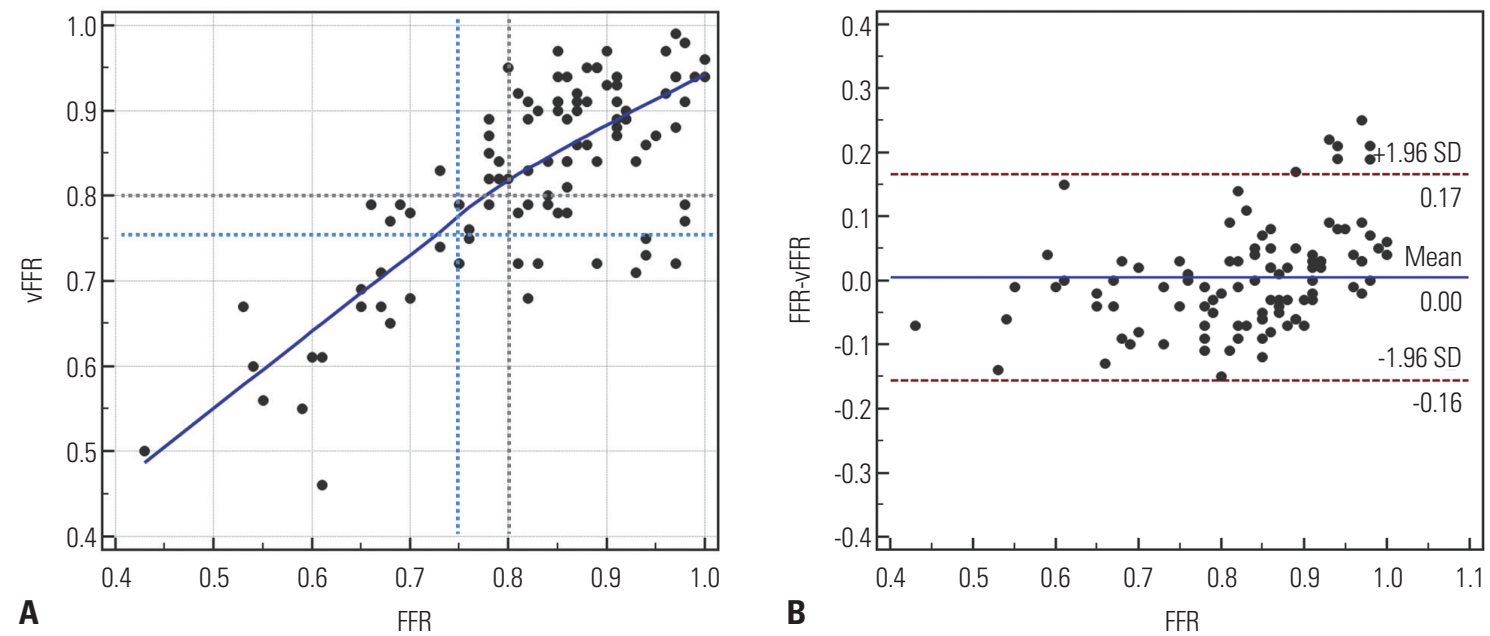

Fig. 3. Linear regression (A) and Bland-Altman analysis (B) between vFFR and FFR. Correlation coefficient ( $\mathrm{r}$ ) between vFFR and FFR was $0.75(95 \% \mathrm{CI} 0.65$ to 0.83 ), and Bland-Altman analysis showed a mean bias of 0.005 ( $95 \% \mathrm{CI}-0.011$ to 0.021 ), with $95 \%$ limits of agreement of -0.16 to 0.17 . vFFR, virtual fractional flow reserve; $\mathrm{Cl}$, confidence interval.

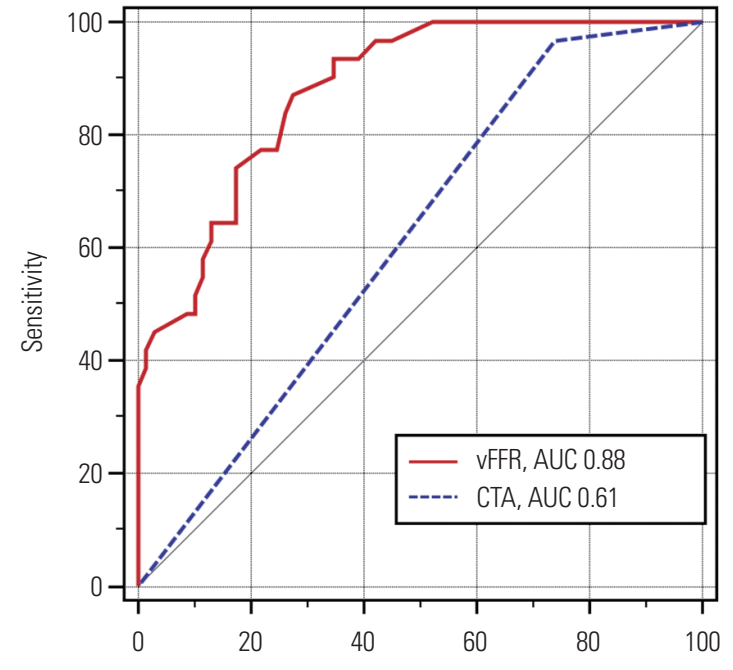

A

100-specificity

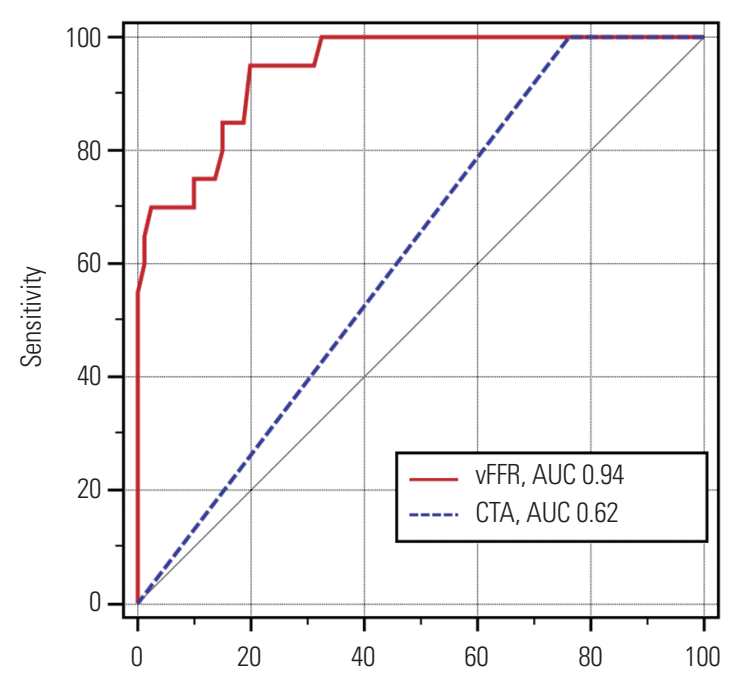

B

100-specificity

Fig. 4. ROC demonstrating AUCs for vFFR and obstructive $(\geq 50 \%)$ CTA stenosis for the discrimination of lesion-specific ischemia using FFR cutoff values of 0.8 and 0.75 . (A) The AUC for vFFR was significantly higher $(0.88,95 \% \mathrm{Cl} 0.80-0.94)$ than CTA $\geq 50 \%$ stenosis $(0.61,95 \% \mathrm{Cl} 0.51-0.71)$ when an FFR cutoff of 0.8 was used. (B) The AUC value for vFFR was excellent (0.94, 95\% Cl $0.88-0.98)$, compared to the CTA $\geq 50 \%$ stenosis $(0.62,95 \% \mathrm{Cl} 0.52-0.71)$, when an FFR cutoff of 0.75 was used. ROC, receiver operating characteristic curve; AUC, areas under receiver operating characteristic curve; vFFR, virtual fractional flow reserve; $\mathrm{CTA}$, com-puted tomography angiography; $\mathrm{Cl}$, confidence interval.

and NPV of $87.0 \%$ (95\% CI 77.0-95.7), 95.0\% (95\% CI 75.199.9), $80.0 \%$ (95\% CI 69.6-88.1), 54.3\% (95\% CI 43.1-65.1), and $98.5 \%$ (95\% CI 90.4-99.8), respectively. In addition, the AUC value for vFFR was excellent [0.94 (95\% CI 0.88-0.98) vs. 0.62 (95\% CI 0.52-0.71)], compared with CTA $\geq 50 \%$ stenosis (Fig. 4B). Fig. 5 shows the comparison of the diagnostic performances between vFFR and FFR at 0.8 , between vFFR and FFR at 0.75 , and between CTA $\geq 50 \%$ stenosis and FFR at 0.8 .

The diagnostic performances according to vessel size were also evaluated by separating large vessels $(n=70)$, such as the $\mathrm{LM}, \mathrm{LAD}$, and RCA, from small vessels $(\mathrm{n}=30)$, such as $\mathrm{LCX}, \mathrm{DG}$, $\mathrm{OM}$, and RI. Large vessels showed higher accuracy than small vessels both with FFR cutoffs of 0.80 ( $80.5 \%$ vs. $73.3 \%)$ and 0.75
(88.9\% vs. $83.3 \%)$.

\section{DISCUSSION}

In the present study, we developed a novel non-invasive CTAderived on-site vFFR method and demonstrated its excellent correlation with invasively measured FFR. In addition, this method exhibited excellent diagnostic performance for detecting ischemia producing lesions, compared to invasive FFR as a reference standard. Furthermore, vFFR had significantly higher diagnostic performance and improved discriminatory power for the detection of lesion-specific ischemia, compared 


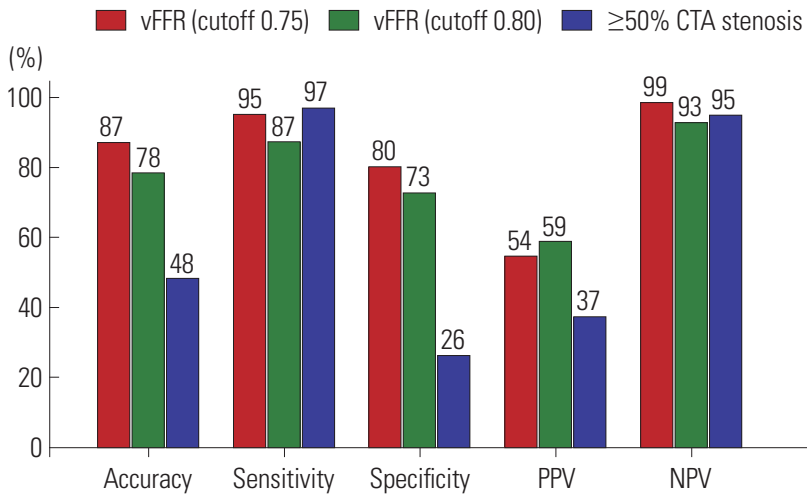

Fig. 5. Diagnostic performance of vFFR using a cutoff of 0.75 (red), vFFR using a cutoff of 0.8 (green), and obstructive ( $\geq 50 \%)$ CTA stenosis (blue) for lesion-specific ischemia detection. vFFR, virtual fractional flow reserve; CTA, computed tomography angiography.

to obstructive ( $\geq 50 \%$ ) CTA stenosis alone. Moreover, we also discovered that when an FFR cutoff of 0.75 was used, the diagnostic accuracy and discriminatory ability of vFFR for ischemia detection was greatly enhanced.

CTA is a robust noninvasive tool for ruling out obstructive or high-grade coronary artery stenosis, obviating unnecessary use of invasive catheterization. ${ }^{4-7}$ On the other hand, CTA's tendency to overestimate coronary artery stenosis can lead to additional testing that is often unnecessary due to its low specificity and PPV for the detection of ischemia causing stenosis. ${ }^{8,9}$ Based on these limitations, FFR-CT was created, and several FFR-CT studies have shown great improvement in the diagnostic performance of ischemia detection by increasing specificity and PPV compared with other non-invasive imaging modalities, such as stress echocardiography, single photon emission computed tomography, or cardiac magnetic resonance. ${ }^{10-12,28}$ However, FFR-CT has several major limitations, including high addon costs (approximately \$1500), potential risk of exposure of personal patient information based on need for off-site postprocessing, and considerable processing time leading to a potential delay in patient care. ${ }^{14-18}$ Furthermore, a recently introduced on-site CTA derived cFFR (Siemens cFFR, version 1.4; Siemens Healthcare, Forchheim, Germany) has been shown to have high diagnostic accuracy. ${ }^{29}$ However, this method requires a semi-automated coronary lumen segmentation process (-60 minutes per case), which might be a considerable limitation when considering its application to daily practice. ${ }^{30}$

On the other hand, the vFFR does not require the use of a supercomputer to compute coronary flow. Instead, the implementation of on-site CFD calculation is done using a novel parallel computing method. This method is based on a cluster with 40 cores, which then decomposes the domain into 40 subdomains and assigns a sub-domain to each computing core. Additionally, the coronary lumen segmentation and 3D reconstruction is conducted by a completely automated algorithm without any operator intervention, thus making the results available within 30 minutes, including segmentation and CFD calculation. In addition, the median radiation dose of CTA in this study was $3.92 \mathrm{mSv}$ (95\% CI 2.56 to 5.65), and 9 patients (16\%) received less than $1 \mathrm{mSv}$ (using $80 \mathrm{kVp}$ along prospective electrocardiogram gating). To date, several studies have shown that CTA is able to visualize not only stenosis severity but also adverse plaque characteristics, which are closely related with future adverse events, in addition to lesion specific ischemia. ${ }^{31-35}$ For example, Gaur, et al. ${ }^{36}$ demonstrated that adverse plaque characteristics provide additive valve in FFR-CT for prediction of ischemia. Based on these findings, CTA with the aid of on-site vFFR could potentially provide a comprehensive evaluation of coronary stenosis and adverse plaque features, as well as assessment of hemodynamically significant CAD, which could lead to a true "one-stop shop" with sub milli-Sievert radiation exposure.

This study is not without limitations. First, this was a retrospective pioneering study with a limited number of cases. Presently, vFFR requires further well-powered validation in prospective multicenter trials. Second, the vessels interrogated by FFR were limited to those clinically indicated, introducing potential selection bias. Lastly, we were unable to evaluate $5 \%$ (3/60) of patients in whom vessels were heavily calcified or there were severe motion artifacts that made CTA-derived FFR methods impossible. CTA image quality was also an important factor for successful simulation in this study, which is the case in most other imaging studies. ${ }^{37,38}$ Recently developed high spatial resolution and wider CTA detector coverage may provide a solution for this issue in the near future. ${ }^{39}$

In conclusion, a novel on-site vFFR computing system employing a fully automated segmentation algorithm using a parallel computation method showed excellent diagnostic performance for the detection of lesion-specific ischemia and was significantly faster and cheaper.

\section{ACKNOWLEDGEMENTS}

This work was supported by the Institute for Information \& Communications Technology Promotion (IITP) grant funded by the Korea government (MSIP) (No. R0101-16-0171, Development of Multi-modality Imaging and 3D Simulation-Based Integrative Diagnosis-Treatment Support Software System for Cardiovascular Diseases).

\section{AUTHOR CONTRIBUTIONS}

Conceptualization: Hyung-Bok Park, Sang-Wook Lee, and Hyuk-Jae Chang. Data curation: Hyung-Bok Park, Yeonggul Jang, Sang-Wook Lee, and Hyuk-Jae Chang. Formal analysis: Hyung-Bok Park, Yeonggul Jang, and Sang-Wook Lee. Funding acquisition: Hyuk-Jae Chang. Investigation: Hyung-Bok Park, Yeonggul Jang, Sang-Wook Lee, Reza Arsanjani, Minh Tuan Nguyen, Sang-Eun Lee, Byunghwan Jeon, Sunghee Jung, and Seongmin Ha. Methodology: Hyung-Bok Park, Sang-Wook Lee, and Hyuk-Jae Chang. Project administration: HyukJae Chang. Resources: Hyuk-Jae Chang and Sang-Wook Lee. Software: 
Yeonggul Jang, Reza Arsanjani, Minh Tuan Nguyen, and Youngtaek Hong. Supervision: Sang-Wook Lee and Hyuk-Jae Chang. Validation: Yeonggul Jang, Reza Arsanjani, Minh Tuan Nguyen, and Sang-Eun Lee. Visualization: Seongmin Ha and Sekeun Kim. Writing-original draft: Hyung-Bok Park, Yeonggul Jang, Sang-Wook Lee, and Hyuk-Jae Chang. Writing_-review \& editing: Hyung-Bok Park, Yeonggul Jang, Sang-Wook Lee, and Hyuk-Jae Chang. Approval of final manuscript: All authors.

\section{ORCID iDs}

Hyung-Bok Park
Yeonggul Jang
Reza Arsanjani
Minh Tuan Nguyen
Sang-Eun Lee
Byunghwan Jeon
Sunghee Jung
Youngtaek Hong
Seongmin Ha
Sekeun Kim
Sang-Wook Lee
Hyuk-Jae Chang

https://orcid.org/0000-0002-3773-2665 https://orcid.org/0000-0002-5805-7494 https://orcid.org/0000-0001-7081-4286 https://orcid.org/0000-0002-2915-9133 https://orcid.org/0000-0001-6645-4038 https://orcid.org/0000-0002-0414-1762 https://orcid.org/0000-0002-8042-457X https://orcid.org/0000-0003-2104-5905 https://orcid.org/0000-0002-0731-2301 https://orcid.org/0000-0003-4196-6242 https://orcid.org/0000-0002-8600-9991 https://orcid.org/0000-0002-6139-7545

\section{REFERENCES}

1. Tonino PA, De Bruyne B, Pijls NH, Siebert U, Ikeno F, van' t Veer M, et al. Fractional flow reserve versus angiography for guiding percutaneous coronary intervention. N Engl J Med 2009;360:213-24.

2. Pijls NH, Fearon WF, Tonino PA, Siebert U, Ikeno F, Bornschein B, et al. Fractional flow reserve versus angiography for guiding percutaneous coronary intervention in patients with multivessel coronary artery disease: 2-year follow-up of the FAME (Fractional Flow Reserve Versus Angiography for Multivessel Evaluation) study. J Am Coll Cardiol 2010;56:177-84.

3. De Bruyne B, Pijls NH, Kalesan B, Barbato E, Tonino PA, Piroth Z, et al. Fractional flow reserve-guided PCI versus medical therapy in stable coronary disease. N Engl J Med 2012;367:991-1001.

4. Budoff MJ, Dowe D, Jollis JG, Gitter M, Sutherland J, Halamert E, et al. Diagnostic performance of 64-multidetector row coronary computed tomographic angiography for evaluation of coronary artery stenosis in individuals without known coronary artery disease: results from the prospective multicenter ACCURACY (Assessment by Coronary Computed Tomographic Angiography of Individuals Undergoing Invasive Coronary Angiography) trial. J Am Coll Cardiol 2008;52:1724-32.

5. Miller JM, Rochitte CE, Dewey M, Arbab-Zadeh A, Niinuma H, Gottlieb I, et al. Diagnostic performance of coronary angiography by 64-row CT. N Engl J Med 2008;359:2324-36.

6. Meijboom WB, Meijs MF, Schuijf JD, Cramer MJ, Mollet NR, van Mieghem CA, et al. Diagnostic accuracy of 64-slice computed tomography coronary angiography: a prospective, multicenter, multivendor study. J Am Coll Cardiol 2008;52:2135-44.

7. Dewey M, Rief M, Martus P, Kendziora B, Feger S, Dreger H, et al. Evaluation of computed tomography in patients with atypical angina or chest pain clinically referred for invasive coronary angiography: randomised controlled trial. BMJ 2016;355:i5441.

8. Raff GL, Gallagher MJ, O'Neill WW, Goldstein JA. Diagnostic accuracy of noninvasive coronary angiography using 64-slice spiral computed tomography. J Am Coll Cardiol 2005;46:552-7.

9. Leber AW, Knez A, von Ziegler F, Becker A, Nikolaou K, Paul S, et al. Quantification of obstructive and nonobstructive coronary le- sions by 64-slice computed tomography: a comparative study with quantitative coronary angiography and intravascular ultrasound. J Am Coll Cardiol 2005; 46:147-54.

10. Koo BK, Erglis A, Doh JH, Daniels DV, Jegere S, Kim HS, et al. Diagnosis of ischemia-causing coronary stenoses by noninvasive fractional flow reserve computed from coronary computed tomographic angiograms. Results from the prospective multicenter DISCOVER-FLOW (Diagnosis of Ischemia-Causing Stenoses Obtained Via Noninvasive Fractional Flow Reserve) study. J Am Coll Cardiol 2011;58:1989-97.

11. Min JK, Leipsic J, Pencina MJ, Berman DS, Koo BK, van Mieghem C, et al. Diagnostic accuracy of fractional flow reserve from anatomic CT angiography. JAMA 2012;308:1237-45.

12. Nørgaard BL, Leipsic J, Gaur S, Seneviratne S, Ko BS, Ito H, et al. Diagnostic performance of noninvasive fractional flow reserve derived from coronary computed tomography angiography in suspected coronary artery disease: the NXT trial (analysis of coronary blood flow using CT angiography: next steps). J Am Coll Cardiol 2014;63:1145-55.

13. Ding A, Qiu G, Lin W, Hu L, Lu G, Long X, et al. Diagnostic performance of noninvasive fractional flow reserve derived from coronary computed tomography angiography in ischemia-causing coronary stenosis: a meta-analysis. Jpn J Radiol 2016;34:795-808.

14. Min JK, Taylor CA, Achenbach S, Koo BK, Leipsic J, Nørgaard BL, et al. Noninvasive fractional flow reserve derived from coronary CT angiography: clinical data and scientific principles. JACC Cardiovasc Imaging 2015;8:1209-22.

15. Kimura T, Shiomi H, Kuribayashi S, Isshiki T, Kanazawa S, Ito H, et al. Cost analysis of non-invasive fractional flow reserve derived from coronary computed tomographic angiography in Japan. Cardiovasc Interv Ther 2015;30:38-44.

16. Douglas PS, De Bruyne B, Pontone G, Patel MR, Norgaard BL, Byrne RA, et al. 1-year outcomes of FFRCT-guided care in patients with suspected coronary disease: the PLATFORM study. J Am Coll Cardiol 2016;68:435-45.

17. Rajani R, Modi B, Ntalas I, Curzen N. Non-invasive fractional flow reserve using computed tomographic angiography: where are we now and where are we going? Heart 2017;103:1216-22.

18. Otake H, Taylor CA, Matsuo H, Tanaka N, Akasaka T. Noninvasive fractional flow reserve derived from coronary computed tomography angiography-is this just another new diagnostic test or the long-awaited game changer? Circ J 2017;81:1085-93.

19. Jeon B, Hong Y, Han D, Jang Y, Jung S, Hong Y, et al. Maximum a posteriori estimation method for aorta localization and coronary seed identification. Pattern Recognition 2017;68:222-32.

20. Han D, Shim H, Jeon B, Jang Y, Hong Y, Jung S, et al. Automatic coronary artery segmentation using active search for branches and seemingly disconnected vessel segments from coronary CT angiography. PLoS One 2016;11:e0156837.

21. Jang Y, Kim DH, Jeon B, Han D, Shim H, Chang HJ. Generation of triangular mesh of coronary artery using mesh merging. Journal of KIISE 2016;43:419-29.

22. Sankaran S, Esmaily Moghadam M, Kahn AM, Tseng EE, Guccione JM, Marsden AL. Patient-specific multiscale modeling of blood flow for coronary artery bypass graft surgery. Ann Biomed Eng 2012;40:2228-42.

23. Sharma P, Itu L, Zheng X, Kamen A, Bernhardt D, Suciu C, et al. A framework for personalization of coronary flow computations during rest and hyperemia. Conf Proc IEEE Eng Med Biol Soc 2012; 2012:6665-8.

24. Murray CD. The physiological principle of minimum work: I. The vascular system and the cost of blood volume. Proc Natl Acad Sci U S A 1926;12:207-14. 
25. Nguyen MT, Jeon BJ, Chang HJ, Lee SW. Domain decomposition based parallel computing for multi-scale coronary blood flow simulations. Computers \& Fluids 2019;191:104254.

26. Karypis G, Kumar V. A fast and high quality multilevel scheme for partitioning irregular graphs. SIAM J Sci Comput 1998;20:359-92.

27. DeLong ER, DeLong DM, Clarke-Pearson DL. Comparing the areas under two or more correlated receiver operating characteristic curves: a nonparametric approach. Biometrics 1988;44:837-45.

28. Nørgaard BL, Jensen JM, Leipsic J. Fractional flow reserve derived from coronary CT angiography in stable coronary disease: a new standard in non-invasive testing? Eur Radiol 2015;25:2282-90.

29. Renker M, Schoepf UJ, Wang R, Meinel FG, Rier JD, Bayer RR 2nd, et al. Comparison of diagnostic value of a novel noninvasive coronary computed tomography angiography method versus standard coronary angiography for assessing fractional flow reserve. Am J Cardiol 2014;114:1303-8.

30. Yang DH, Kim YH, Roh JH, Kang JW, Ahn JM, Kweon J, et al. Diagnostic performance of on-site CT-derived fractional flow reserve versus CT perfusion. Eur Heart J Cardiovasc Imaging 2017;18:43240.

31. Motoyama S, Kondo T, Sarai M, Sugiura A, Harigaya H, Sato T, et al. Multislice computed tomographic characteristics of coronary lesions in acute coronary syndromes. J Am Coll Cardiol 2007;50: 319-26.

32. Motoyama S, Sarai M, Harigaya H, Anno H, Inoue K, Hara T, et al. Computed tomographic angiography characteristics of atherosclerotic plaques subsequently resulting in acute coronary syndrome. J Am Coll Cardiol 2009;54:49-57.

33. Motoyama S, Ito H, Sarai M, Kondo T, Kawai H, Nagahara Y, et al.
Plaque characterization by coronary computed tomography angiography and the likelihood of acute coronary events in mid-term follow-up. J Am Coll Cardiol 2015;66:337-46.

34. Park HB, Heo R, Ó Hartaigh B, Cho I, Gransar H, Nakazato R, et al. Atherosclerotic plaque characteristics by CT angiography identify coronary lesions that cause ischemia: a direct comparison to fractional flow reserve. JACC Cardiovasc Imaging 2015;8:1-10.

35. Nakazato R, Park HB, Gransar H, Leipsic JA, Budoff MJ, Mancini $\mathrm{GB}$, et al. Additive diagnostic value of atherosclerotic plaque characteristics to non-invasive FFR for identification of lesions causing ischaemia: results from a prospective international multicentre trial. EuroIntervention 2016;12:473-81.

36. Gaur S, Øvrehus KA, Dey D, Leipsic J, Bøtker HE, Jensen JM, et al. Coronary plaque quantification and fractional flow reserve by coronary computed tomography angiography identify ischaemiacausing lesions. Eur Heart J 2016;37:1220-7.

37. Leipsic J, Yang TH, Thompson A, Koo BK, Mancini GB, Taylor C, et al. CT angiography (CTA) and diagnostic performance of noninvasive fractional flow reserve: results from the Determination of Fractional Flow Reserve by Anatomic CTA (DeFACTO) study. AJR Am J Roentgenol 2014;202:989-94.

38. Nørgaard BL, Gaur S, Leipsic J, Ito H, Miyoshi T, Park SJ, et al. Influence of coronary calcification on the diagnostic performance of CT angiography derived FFR in coronary artery disease: a substudy of the NXT trial. JACC Cardiovasc Imaging 2015;8:1045-55.

39. Nerlekar N, Ko BS, Nasis A, Cameron JD, Leung M, Brown AJ, et al. Impact of heart rate on diagnostic accuracy of second generation 320-detector computed tomography coronary angiography. Cardiovasc Diagn Ther 2017;7:296-304. 